\title{
Body image and the relation to mindfulness and self-compassion in physical education students: a cross-cultural study
}

\author{
Petra Jansen, ${ }^{1}$ Kashef Zayed, ${ }^{2}$ Jürgen Kittsteiner ${ }^{1}$ \\ ${ }^{1}$ Faculty of Human Science, University of Regensburg, Regensburg, Germany; ${ }^{2}$ Department of \\ Physical Education \& Sport Sciences, Sultan Quaboos University, Muscat, Sultanate of Oman
}

\begin{abstract}
The present study investigated the body images of Omani and German physical education students. 199 students completed a body image test, a dispositional mindfulness and a self-compassion measurement, as well as a short physical activity questionnaire. Overall, our results demonstrate that females showed a higher body distortion than males and students in Germany displayed a higher body distortion effect. Concerning mindfulness and self-compassion, students from Oman showed higher values
\end{abstract}

Correspondence: Petra Jansen, Faculty of Human Science, University of Regensburg, Regensburg, Germany.

Tel.: +49.941.9432518.

E-mail: petra.jansen@ur.de

Key words: Self-kindness; students; mindfulness; BMI; body image, body dissatisfaction.

Acknowledgments: The authors thank the students for their participation.

Contributions: PJ designed the study, analyzed the data and wrote the first draft of the paper. KZ helped designing the study. He organized data acquisition, helped analyzing the data and discussed the first draft of the paper. JK acquired the data and discussed the first draft of the paper.

Conflict of interest: The authors declare that the research was conducted in the absence of any commercial or financial relationships that could be construed as a potential conflict of interest.

Funding: None.

Ethical approval: All procedures performed in studies involving human participants were in accordance with the ethical standards of the institutional and/or national research committee and with the 1964 Helsinki declaration and its later amendments or comparable ethical standards.

Availability of data and materials: Data and materials available from the corresponding author.

Received for publication: 8 June 2020.

Revision received: 22 July 2020.

Accepted for publication: 23 Novembrer 2020.

This work is licensed under a Creative Commons AttributionNonCommercial 4.0 International License (CC BY-NC 4.0).

(C) Copyright: the Author(s), 2020

Licensee PAGEPress, Italy

Health Psychology Research 2020; 8:9172

doi:10.4081/hpr.2020.9172 than German students in self-compassion, but not in the dispositional mindfulness measurement. However, self-compassion and mindfulness were not related to the body distortion effect. This is the first study that provides unique data of self-compassion and mindfulness in an Arabic sample, and shows that self-compassion is higher in Omani students.

\section{Introduction}

Body image in Omani and German physical education students

The years at university are a lifetime that present a transition from adolescence to adulthood. This transition is accompanied by decreases in physical activity and weight gain (Nelson, Story, Larson, Neumark-Sztainer, \& Lytle, 2008), which changes the body image of students. Body image is defined as the picture of one's own body consisting of an attitudinal and a perceptual component, which means that elements relate to body shape (Paap \& Gardner, 2011). It has been shown that females have a poorer body image than males (El Ansari, Dibby, \& Stock, 2014; Feingold \& Mazzella, 1998) and that especially adolescent girls associated body dissatisfaction with the concept of self-esteem (Furnham, Badmin, \& Sneade, 2002). Concerning the evaluation of the own body in students in several European countries, including Germany, it was demonstrated that weight ideals are rather uniform across European countries: female students more often find themselves too fat even though they have a normal BMI and male students more often perceive themselves as "too thin" (Mikolajczyk et al., 2010).

Regarding the body image in university students in Oman there are only limited studies and results: in a study with female university students from five Arabic countries, 249 women from Oman participated. Of them, $62 \%$ were satisfied with their body, and only $22 \%$ dieted in the past 6 months to lose weight. This was the lowest rate among female students in the five Arabic countries. Seventeen percent of Omani female university students recognized that they would like to have a body shape similar to Western fashion models, and $58 \%$ of the Omani female students believed that men in their country preferred slim women (Musaiger, 2015).

\section{Body image and the relation to mindfulness and self- compassion}

Mindfulness is defined as the ability to be present in each moment without judging the situation (Crane, 2002). Mindfulness training can reduce symptoms of stress and negative feelings and also mood disturbances (Brown \& Ryan, 2003). Self-compassion describes the ability to be kind to the own person when one is confronted with personal failures. The three important elements of 
self-compassion are self-kindness instead of self-judgment, mindfulness instead of over-identification and common humanity instead of isolation (Neff, 2003).

Because body image distress is so common among Western university students, and since about 50\% admitted that body shape had a moderate influence on their self-worth (Cain, Epler, Steinley, \& Sher, 2010), it is necessary to search for appropriate programs to avoid such body image distress. Thus, it could be shown that a writing based intervention with elements of self-compassion may decrease body image concerns (Seekis, Bradley, \& Duffy, 2017) and for example appearance dissatisfaction (Moffitt, Neumann, \& Williamson, 2018). Individuals who were more mindful engaged less often in body comparison. Also the authors found a small mediation effect for mindfulness in the relation of body comparison and body satisfaction (Dijkstra \& Barelds, 2011). There was also a positive relationship between self-compassion and selfesteem in female college students' body image. When both aspects, self-compassion and self-esteem were included as predictors, selfcompassion accounted for unique variance in body preoccupation (Wasylkiw, MacKinnon, \& MacLellan, 2012).

\section{Goal of the study}

The goals of the study are twofold: first of all, it should be investigated if there are differences in body image, dispositional mindfulness and self-compassion in German and Omani female and male students of physical education. Students from Oman were chosen as one example of an Arabic country because this Arabic country has gone through a rapid economic development. According to the Central Intelligence Agency (2017 estimates) the gross domestic product (at purchasing power parity) per capita is comparable between both countries: (Oman: 45.500\$ and Germany: 50.200\$) (https://en.wikipedia.org/wiki/List_of_countries_by_GDP_(PPP)_per_capita retrieved the 16th of december 2020). Therefore, we assumed that it is possible to adapt the tests, which were constructed for people living in the West, for the Arabic students. Second, we wanted to investigate if dispositional mindfulness and self-compassion relate to the body image of students in Germany and Oman in the same manner. A possible difference might be due to the different religions, around $85 \%$ of the inhabitants in Oman are Muslims: Mussap (2009) showed that in a sample with 98 Muslim and 91 non-Muslim Australian women that the religious faith was inversely related to body dissatisfaction.

\section{Hypotheses}

i) According to El Ansarie et al. (2015) we expect a gender difference in body image in both countries with males having a better body image than females. According to Musaiger (2015) we expect that the body images of the Omani female students are better than the one of the German students.

ii) Until now, there are no data comparing dispositional mindful- ness and self-compassion in an Arabic culture compared to a Western one. For this it remains to be investigated if there are differences between both cultures.

iii) We want to investigate if a body distortion relates to mindfulness and self-compassion in both cultures in the same manner.

\section{Materials and Methods}

\section{Participants}

99 students (49 males and 50 females, mean age $=21.10$ years, $\mathrm{SD}=2.22$ ) from the same university in Germany, and 100 students from the same university in Oman (49 males and 51 females, mean age $=20.76$ years, $\mathrm{SD}=1.37$ ) participated. All students were physical education majors. All participants gave their written consent for participation and the experiment was conducted according to the ethical guidelines of the Helsinki declaration. They were tested in groups up to 20 participants. Each test session lasted around 30 minutes. Table 1 shows the demographic data for the German and the Omani group for females and males separately.

\section{Materials}

\section{Mindfulness Attention Awareness Scale}

The Mindfulness Attention Awareness Scale (MAAS) consists of 15 items, which investigate the dispositional mindfulness as a one dimensional structure (Brown \& Ryan, 2003). Items are rated on a 6-point Likert scale from 1 (almost always) to 6 (almost never), a mean score is calculated. Cronbach's Alpha ranged between .80 and .90 in the German version of the MAAS (Michalak, Heidenreich, Ströhle, \& Nachtigall, 2008). The MAAS is useful in participants without meditation experience.

\section{Self-Compassion}

The construct of self-compassion was examined with the SelfCompassion Scale (Neff, 2003) including the following six elements: self-kindness, reduced self-judgment, common humanity, reduced isolation, mindfulness and reduced over-identification. Responses had to be given on a scale from 1 (almost never) and 5 (almost always). The items self-kindness, common humanity and mindfulness were positively associated, and the items self-judgment, isolation and over-identification are negatively related. The overall score reached a Cronbach's alpha $=.92$ and the Cronbach's alpha of the six subscales varied between .80 and .88 . New results support the use of one total score as well as the six subscales (Neff, Tóth-Király, Yarnell, Arimitsu, \& Castilho, 2018).

\section{Body Image Assessment, BIAS-BD (Gardner, Jappe, \& Gardner, 2009)}

The scale consisted of 17 female or 17 male contour line draw-

Table 1. Means (standard deviations) for age and BMI in Germany and Oman.

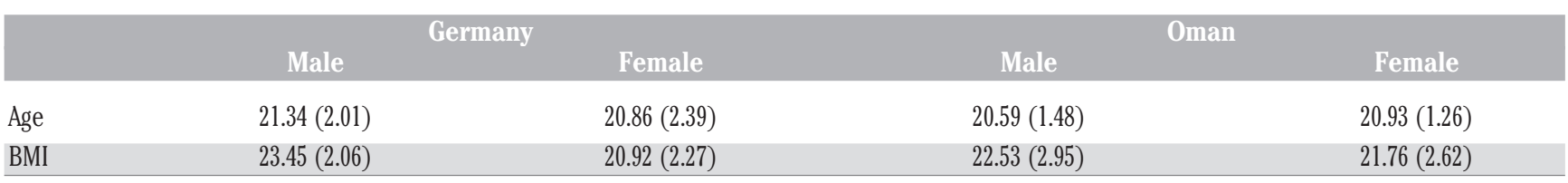


ings which used anthropometric body dimensions of shoulder, chest, waist, hip breadth, thigh breadth and upper leg breadth, see Figure 1. The drawings were constructed for BMI values ranging from $60 \%$ below and $140 \%$ above average. Corresponding BMI values for males were 16.68 - 38.92 and 16.92 - 39.48 for females. Participants had to select their actual (marking with an A) and their ideal size (marking with an I). Body-size distortion was determined by comparing the self-reported BMI with the BMI values that correspond to the actual marked figure. In the study of Gardner et al. (2009) the difference was 2.93 BMI points for females and 0.69 BMI points for males. Test-retest reliability was $\mathrm{r}=0.86$ for actual perceived size, and $\mathrm{r}=0.72$ for ideal size. Concurrent validity was measured as the correspondence between perceived and reported size and was $\mathrm{r}=0.76$.

\section{Questionnaire Physical Activity, RAPA}

Physical activity was measured with the rapid assessment of physical activity which is an easy-to-use valid measure of physical activity in different populations (Topolski et al., 2006). It includes nine questions regarding the range of levels of physical activity in different types of activity. The total score of the first seven items can range between 1 and 7 . The last two items concerning strength training and flexibility or both can be scored separately but were not considered here.

\section{Procedure}

The study took place in April 2018 in Germany and Oman in lessons for physical education students. First, information about the study was given to the students and they were asked to provide their demographic information (age, gender, height and weight). After this, they completed in the following order the RAPA, the BIAS-BD, SCS and MAAS. The whole procedure lasted around 30 minutes. For the Omani students the tests were given in Arabic and for the German students in German.

\section{Statistical analysis}

For the BMI, the physical activity score, the MAAS, SCS total score and the body-distortion score, three separate univariate analyses with the factors "country" and "gender" were conducted. Furthermore for the subscales of the BIAS multivariate analysis of variance with the same factors "country" and "gender" were run. Furthermore, three correlations between mindfulness, self-compassion and the body-distortion effect and second separate analyses of variance were calculated.

\section{Results}

\section{Body mass index}

We registered their BMI. Nine students from Oman did not give any information concerning their weight and height. There was a significant difference for the factor gender, $F(1,186)=31.32$, $\mathrm{p}<0.001, \eta_{\mathrm{p}}{ }^{2}=0.144$ but not for the factor country, $F(1,186)=1.29$, $p=0.257, \eta_{\mathrm{p}}{ }^{2}=0.007$ nor for the interaction between both factors, $F(1,186)=1.93, p=0.166, \eta_{\mathrm{p}}^{2}=0.01$

\section{Physical activity}

Concerning the overall measurement of physical activity, analysis of variance with the factors "country" and "gender" showed no main effect for the factor country, $F(1,195)=1.31$, $p=0.254, \eta_{\mathrm{p}}{ }^{2}=0.007$, neither gender, $F(1,195)=0.228, p=0.634$, $\eta_{\mathrm{p}}{ }^{2}=0.001$, nor an interaction between both factors, $F(1,195)=1.066, p=0.303, \eta_{\mathrm{p}}{ }^{2}=0.005$. The mean score for the German students was $M=6.26(S D=1.25)$ and for the Omani students $M=6.050(S D=1.134)$.

\section{Body image assessment}

The multivariate analysis with the assessment of the actual and the wished figure using Pillai's trace showed a significant effect of "country", $F(2,192)=5.005, p=0.008, \eta_{\mathrm{p}}{ }^{2}=0.050$ and "gender", $F(2,192)=7.78, p=0.001, \eta_{\mathrm{p}}{ }^{2}=0.075$ but no interaction between both factors, $F(2,192)=02.38, p=0.095, \eta_{\mathrm{p}}{ }^{2}=0.024$.

Analyzing the assessment of the actual shape, there was a significant effect of "country", $F(1,193)=7.836, p=0.006, \eta_{\mathrm{p}}{ }^{2}=0.039$ but not for "gender", $F(1,193)=1.524, p=0.218, \eta_{\mathrm{p}}{ }^{2}=0.008$ nor for an interaction between both factors, $F(1,193)=0.495, p=0.483$, $\eta_{\mathrm{p}}{ }^{2}=0.003$. The values were lower for the students from Oman $(M=79.36, S D=15.05)$ compared to the German students $(M=85.02, S D=13.22)$, see Figure 2A.

The assessment of the actual shape was transformed to the corresponding BMI for each gender and country. German males assessed $\mathrm{BMI}=24.18$ (real: 23.45), German females assessed $\mathrm{BMI}=23.41$; Oman males assessed $\mathrm{BMI}=22.21$ (real: 22.53), Oman females assessed $\mathrm{BMI}=22.23$ (real: 21.01). For each gender

A)

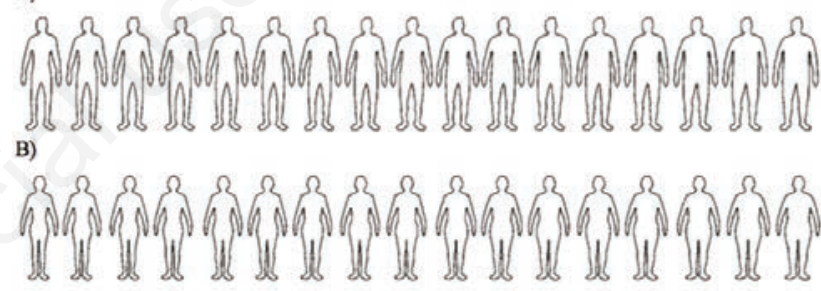

Figure 1. Silhouettes of male (A) and female (B) drawings.

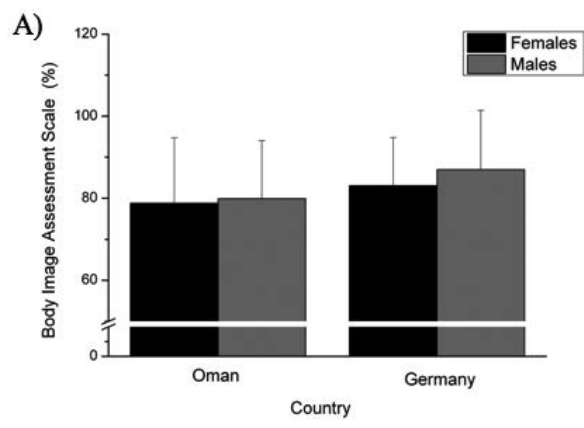

B)

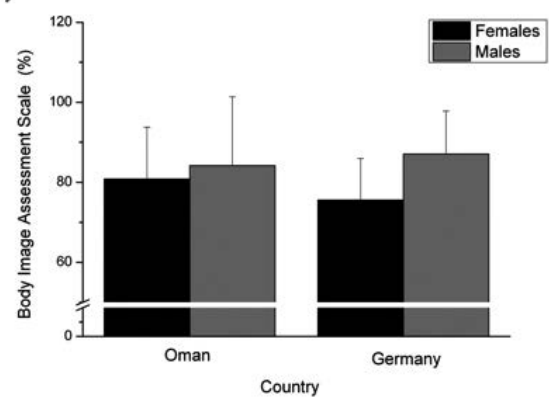

Figure 2. Means (standard deviations) for actual (A) and wished shape (B) dependent on country and gender. 
and country there was a high correlation between the real and assessed BMI between $r=0.597$ and $r=0.822$. This is in line with the study of Gardner et al. (2009) who showed a correlation of $\mathrm{r}=0.76$.

Regarding the assessment of the wished shape, there was significant effect of "gender", $F(1,193)=15.62, p<0.001, \eta_{\mathrm{p}}{ }^{2}=0.075$ but not for "country", $F(1,193)=0.402, p=0.527, \eta_{\mathrm{p}}{ }^{2}=0.002$. However, there was an interaction between both factors, $F(1,193)=4.78, p=0.030, \eta_{\mathrm{p}}{ }^{2}=0.024$. For the male students there was no significant difference between Omani and German students $t(98)=0.994, p=0.323$ but for the female students the results indicated a significant difference, $t(98)=-2.239, p=0.029$. The wished shape for the German female students was lower in percentage than the ones for the Omani female students, see Figure 2B.

In accordance to Gardner et al. (2009) actual and wished size are correlated $(\mathrm{r}=0.286)$, indicating that if the actual size increases the wished size also increases. The analysis of body size distortion (real BMI-assessed actual BMI) indicated a main effect of "country", $\quad F(1,184)=9.033, \quad p=0.003, \quad \eta_{\mathrm{p}}{ }^{2}=.047$ and "gender", $F(1,184)=8.29, p=0.004, \eta_{\mathrm{p}}{ }^{2}=0.043$ but no interaction between both factors, $F(1,184)=1.161, p=0.283, \eta_{\mathrm{p}}{ }^{2}=0.006$. The body size distortion was higher in the German student's group $(M=1.65$, $S D=2.96)$ compared to the Oman student group ( $M=0.396$, $S D=2.97)$ and higher in females $(M=1.64, S D=2.58)$ compared to males $(M=0.405, S D=3.32)$.

\section{Mindfulness}

Concerning the overall MAAS-score, analysis of variance with the factors "country" and "gender" showed no main effect for the factor "country", $\mathrm{F}(1,195)=3.05, \mathrm{p}=.082, \eta_{\mathrm{p}}{ }^{2}=.015$, neither "gender", $\mathrm{F}(1,195)=.004, \mathrm{p}=.952, \eta \mathrm{p} 2<.001$, nor an interaction between both factors, $\mathrm{F}(1,195)=.504, \mathrm{p}=.479, \eta_{\mathrm{p}}{ }^{2}=.003$.

\section{Self-compassion}

An analysis of variance with the factors "country" and "gender" for the mean of self-compassion showed only a main effect for the factor "country", $F(1,195)=25.50, p<0.001, \eta_{\mathrm{p}}{ }^{2}=0.116$, but neither for "gender", $F(1,195)=0.507, p=0.477, \eta_{\mathrm{p}}{ }^{2}=.003$, nor an interaction between both factors, $F(1,195)=2.038, p=0.155$, $\eta_{\mathrm{p}}{ }^{2}=0.010$. Students from Germany $(M=3.16, S D=0.52)$ have a lower score than students from Oman $(M=3.50, S D=0.44)$. The subscales of self-compassion for males and females in Oman and Germany are presented in Table 2.

\section{Correlation}

There was no significant correlation between mindfulness and body distortion and self - compassion and body distortion, neither for the whole sample nor for the Omani and German students separately (all $p>0.08$ ).

\section{Discussion}

Overall, our results demonstrated that females showed a higher body distortion than males. German physical education students showed a higher body distortion effect and students from Oman showed better self-compassion, but not in the dispositional mindfulness measurement, MAAS.

\section{Body image in Omani and German physical education students}

In general, the same pattern regarding body image could be revealed in both countries: females showed a higher body distortion effect, or in other words, males showed higher scores of positive aspects of body image. This is in line with former studies (e.g. El Ansali et al., 2014, Feingold \& Mazella, 1998) but for the first time it was demonstrated for students from an Arabic country. This might indicate that the Arabic culture is getting closer to the Western society where the concern about the body appearance is relevant. One reason for this might be that in the Middle-Eastern countries a rise in internet and social media penetration could be observed (Dennis, Martin, \& Wood, 2017) and a maladaptive effect of the use of social networking sites on body image has been demonstrated in a systematic review including 20 studies (Holland \& Tiggemann, 2016). Even though there is no study which compares the body image in people of the Arabic and Western world until now, the convergence from people in the Arabic to the Western world can be reasoned when investigating the topic of eating attitudes which relate to body image: Kayano et al. (2008) showed that participants from Oman demonstrated similar eating attitudes than people from Western countries.

The body image distortion in this study was less than the one reported by Gardner et al. (2009). Females in this study overestimated their actual size by 1.64 compared to 2.93 and males overestimated it by 0.405 compared to $0.69 \mathrm{BMI}$ points. One reason for the different results might be that in the study of Gardner et al. (2009) university students from different subjects participated whereas in the study presented here only students of physical education took part. These students do a lot of sports in their study and also in their daily life as the mean score of $\mathrm{M}=6.156$ on a scale between 1-7 suggested.

\section{Mindfulness and self-compassion in Oman and Germany}

Our results reveal no difference between dispositional mindfulness in students of Oman and Germany, but significant differences in self-compassion. Omani students show better values, which is similar to the results of a study by Ghorbani et al. (2012) who demonstrated the cross-cultural significance of self-compassion while investigating this with Iranian Muslims. Also, Khorami, Moeini, and Ghamarani (2016) demonstrated that self-compassion training increases the self-compassion of nursery students in Iran.

Table 2. Means (standard deviations) for the six subscales of SCS for male and female students in Oman and Germany.

\begin{tabular}{lccccccccccccc} 
& \multicolumn{2}{c}{$\begin{array}{c}\text { Self-kindness } \\
\text { Oman }\end{array}$} & \multicolumn{2}{c}{ Germany } & \multicolumn{2}{c}{ Omandfulness } & \multicolumn{2}{c}{ Humanity } & \multicolumn{2}{c}{ Self-judgment } & \multicolumn{2}{c}{ Isolation } & \multicolumn{2}{c}{ Over-identification } \\
Males & $3.56(0.57)$ & $3.03(0.62)$ & $3.35(0.57)$ & $3.13(0.61)$ & $3.39(0.79)$ & $2.96(0.84)$ & $2.43(0.65)$ & $2.86(1.25)$ & $2.54(0.74)$ & $2.29(0.87)$ & $2.44(0.89)$ & $2.73(0.75)$ \\
Females & $3.57(0.71)$ & $3.09(0.73)$ & $3.39(0.59)$ & $3.14(0.62)$ & $3.61(0.81)$ & $2.99(0.79)$ & $2.43(0.72)$ & $2.95(0.70)$ & $2.61(0.73)$ & $2.74(0.98)$ & $2.36(0.78)$ & $3.01(0.77)$ \\
\hline
\end{tabular}




\section{Body distortion effect in relation to mindfulness and self-compassion in Oman and Germany}

Our results showed that mindfulness and self-compassion are not related to the body distortion effect in Omani and German physical education students. For this, it has to be discussed, if for healthy young physical education students, who trained their body a lot, the concepts of mindfulness and self-compassion are relevant for a possible body image distortion. However, all students were physical education students. On the one hand we assume a higher awareness of bodily functions, on the other hand more concerns might exist. The studies should be repeated with students from other faculties and non-student samples.

\section{Limitations and future studies}

The study is limited by the fact that until now no validated tests concerning the issues investigated are validated in Arabic. The English version of the physical activity measurement and the mindfulness- and self-compassion measurement were translated by the second co-author in Arabic. The body image assessment was a language free measurement.

Furthermore, the choice of both countries is only one example for two nations in the Western and Arab world. Due to their similar GDP per capita the choice seemed to be justified, however the investigation has to be enlarged to other European and Middle East countries to investigate a more pronounce picture of the relation of self-compassion and body image in two different cultures. It has to be acknowledged that Arab-Islamic countries are far from constituting a homogeneous group as European countries.

Another limiting effect is that the concept of body image was investigated in only some aspects. Several other aspects as for example body shame or body surveillance were not investigated (Toole \& Craighead, 2016). Furthermore, the concept of mindfulness should be investigated in more detail according to body image. The MAAS is a widely used questionnaire for the investigation of mindfulness, it only allows investigation of mechanisms involving very specific aspect of mindfulness, namely acting awareness (Coffey \& Hartman, 2008).

Because our study was cross-sectional in nature, the causal relation between for example self-compassion and body image remains quite unclear. Therefore, the effect of self-compassion interventions on body image in both cultures should be investigated. Furthermore, underlying mechanism could be investigated in more detail, as for example cultural values, the role of females in the country and the role of the media in the advertisement of an ideal form of thinness.

\section{Conclusions}

Despite the limitations, we are convinced that our study will contribute to the literature by providing unique data of the dispositional mindfulness and self-compassion as well as the body image in an Arabic and Western sample. Because differences could be carved out in self-compassion and body distortion in both samples the study contributes to the existing knowledge in that way that results from most studies in this research field have to be judged within the cultural context where the data were retrieved. It adds to the existing literature of cross-cultural differences in self-compassion (Neff, Pisitsungkagarn, \& Hsieh, 2008). At least, this study gives a hint to see the Arab-Islamic world not mainly a crisis-laden region (Haller, 2003) but to see the individuals living there, demonstrating a high self-compassion and a higher body satisfaction at least for the sample of students investigated here.

\section{References}

Brown, K. W., \& Ryan, R. M. (2003). The benefits of being present: Mindfulness and its role in psychological well-being. Journal of Personality and Social Psychology, 84, 822-848.

Cain, A. S., Epler, A. J., Steinley, D., \& Sher, K. J. (2010). Stability and change in patterns of concerns related to eating, weight, and shape in young adult women: a latent transition analysis. Journal of Abnormal Psychology, 119, 255-267.

Coffey, K.A., \& Hartman, M. (2008). Mechanisms of action in the inverse relationship between mindfulness and psychological distress. Complementary Health Practice Review, 13, 79-91.

Crane, B. (2002). Full catastrophe living; using the wisdom of your body and mind to face stress, pain and illness. Spirituality and Health International, 3, 52.

Dennis, E.E., Martin, J.D., \& Wood, R. (2017). Media use in the Middle East, 2017: A six-nation survey. Northwestern University in Qatar. Retrieved from www.mediaeastmedia. org/survey/2017.

Dijkstra, P., \& Barelds, D.P.H. (2011). Examining a model of dispositional mindfulness, body comparison, and body satisfaction. Body Image, 8, 419-422.

El Ansari, W., Dibba, E., \& Stock, C. (2014). Body image concerns: levels, correlates and gender differences among students in the United Kingdom. Central European Journal of Public Health, 22, 106-117.

Feingold, A., \& Mazzella, R. (1998). Gender differences in body image are increasing. Psychological Science, 9, 190-195.

Furnham, A., Badmin, N., \& Sneade, I. (2002). Body image dissatisfaction: gender differences in eating attitudes, self-esteem, and reasons for exercise. The Journal of Psychology, 136, 581596.

Gardner, R.M., Jappe, L.M., \& Gardner, L. (2009). Development and validation of a new figural drawing scale for body-image assessment: the BIAS-BD. Journal of Clinical Psychology, 65, $113-122$.

Ghorbani, N., Watson, P.J., Chen, Z., \& Norballa, F. (2012). SelfCompassion in Iranian Muslims: relationships with integrative self-knowledge, mental health, and religious orientation. International Journal for the Psychology of Religion, 22, 106118.

Haller, M. (2003). Europe and the Arab-Islamic World. A sociological perspective on the socio-cultural difference and mutual (mis)perceptions between two neighbouring cultural areas. Innovation, 16(3), 227-252.

Holland, G., \& Tiggemann, M. (2016). A systematic review of the impact of the use of social networking sites on body image and disordered eating outcomes. Body Image, 17, 100-110.

Kayano, M., Yoshiuchi, K., Al-Adawi, S., Viernes, N., Dorvlo, A.S.S., Kumano, H., Kuboki, T., Akabayashi, A. (2008). Eating attitudes and body dissatisfaction in adolescents: Crosscultural study. Psychiatry and Clinical Neurosciences, 62, 1725.

Khorami, E.S., Moeini, M., \& Ghamarani, A. (2016). The effectiveness of self-compassion training: a field Trial. Global Journal of Medicine Researches and Studies, 3, 15-20.

Michalak, J., Heidenreich, T., Ströhle, G., \& Nachtigall, C. (2008). Die deutsche Version der Mindful Attention and Awareness 
Scale (MAAS) Psychometrische Befunde zu einem Achtsamkeitsfragebogen. Zeitschrift für Klinische Psychologie und Psychotherapie, 37, 200-208.

Mikolajczyk, R.T., Maxwell, A.E., El Ansari, W., Stock, C., Petkeviciene, J., \& Guillen-Grima, F. (2010). Relationship between perceived body weight and body mass index based on self- reported height and weight among university students: a cross-sectional study in seven European countries. BMC Public Health, 10, 40.

Moffitt, R.L., Neumann, D.L., \& Williamson, S.P. (2018). Comparing the efficacy of a brief-self-esteem and self-compassion intervention for state body dissatisfaction and self improvement motivation. Body Image, 22, 67-76.

Musaiger, A. (2015). Body weight concern among female university students in five Arab countries - a preliminary cross-cultural study. Annals of Agricultural and Environmental medicine, 22, 349-352.

Mussap, A.J. (2009). Strength of faith and body image in Muslim and non-Muslim women. Mental Health, Religion \& culture, 12 (2), 121-129.

Neff, K. (2003). The development and validation of a scale to measure self-compassion. Self and Identity, 2, 223-250.

Neff, K.D., Pisitsungkagarn, K., \& Hsieh, Y-P. (2008). Self-compassion and self-construal in the United states, Thailand, and Taiwan. Journal of Cross-Cultural Psychology, 39(3), 267-
285.

Neff, K., Tóth-Király, I., Yarnell, L. M., Arimitsu, K., \& Castilho, P. (2018). Examining the factor structure of the self-compassion scale in 20 diverse samples: support for use of a total six subscale scores. Psychological Assessment, 31(1), 27-45.

Nelson, M.C., Story, M., Larson, N.I., Neumark-Sztainer, D., \& Lytle, L. A. (2008). Emerging adulthood and college-aged youth: an overlooked age for weight-related behavior change. Obesity, 16, 2205-2211.

Paap, C.E., \& Gardner, R.M. (2011). Body image disturbance and relationship satisfaction among college students. Personality and Individual Differences, 51, 715-719.

Seekis, V., Bradley, G.H., \& Duffy, A. (2017). The effectiveness of self-compassion and self-esteem writing tasks in reducing body image concerns. Body Image, 23, 206-213.

Toole, A.M., \& Craighead, L.W. (2016). Brief self-compassion meditation training for body image distress in young adult women. Body Image, 19, 104-112.

Topolski, T.D., LoGerfo, J., Patrick, D. L., Williams, B., Walwick, J., \& Patrick, M.M.B.J. (2006). The Rapid Assessment of Physical Activity (RAPA) among older adults. Preventing Chronic Disease, 3(4).

Wasylkiw, L., MacKinnon, A. L., \& MacLellan, A. M. (2012). Exploring the link between self-compassion and body image in university women. Body Image, 9, 236-245. 